\title{
Propensión a aprender de los Adolescentes Infractores de Ley: reflexiones desde el Enfoque Biográfico
}

\author{
Eduardo Sandoval Obando ${ }^{1}$ \\ Universidad Austral de Chile, Valdivia - Chile. \\ Email: eduardo.sandoval.o@gmail.com
}

\begin{abstract}
Resumen: El estudio busca develar la propensión a aprender de cuatro adolescentes infractores de ley derivados al Programa “Salidas Alternativas” de la Región de Los Ríos. Así, nos interesó caracterizar los aprendizajes que éstos construyen, los patrones de su propensión a aprender y los contextos en que ésta se manifiesta, para descubrir alternativas de modificabilidad cognitiva que apoyen las estrategias de reinserción social y educativa. Metodológicamente utilizamos un enfoque interpretativo-cualitativo, recurriendo al análisis de contenido para la interpretación de los datos, siguiendo la lógica de la Teoría Fundamentada (Strauss y Corbin, 2002) y las Historias de Vida (Pujadas, 1992; Bolívar y Otros, 2001). En síntesis, pudimos observar que la propensión a aprender de estos adolescentes se caracteriza por la repetición, autonomía, alta influenciabilidad y esfuerzo, desplegándose notoriamente en contextos informales de aprendizaje; experimentando periodos de progresivo anquilosamiento dentro de la escuela, coartando sus recursos y potencialidades de desarrollo.
\end{abstract}

Palabras clave: Propensión a aprender, adolescente infractor de ley, aprendizaje, modificabilidad cognitiva, historias de vida.

\section{Learning proclivity on Teenage Law Offenders: an analysis from the Biographical Approach}

\begin{abstract}
This study seeks to reveal the propensity to learn in four teenage offenders of law referred to the Program "Alternative Ways Out" on the Los Ríos Region. Thus, it interested us characterizing the learning they develop, the patterns in their propensity to learn and the contexts in which it manifests, in order to discover cognitive modifiability alternatives that can support social and educational reintegration strategies. Methodologically we used an interpretative-qualitative approach, through the analysis of content for the interpretation of the data, following the logic of the Grounded Theory (Strauss and Corbin, 2002) and the Life Histories (Pujadas, 1992; Bolívar et al., 2001). In short, we could observe that the propensity to learn of these adolescents, is characterized by repetition, autonomy, high impressionability and effort; deploying notoriously in informal learning contexts; experiencing periods of progressive ankylosis within school, subverting its resources and development potentials.

Key words: Propensity to learn, law offender adolescent, learning process, cognitive modifiability, life histories.
\end{abstract}




\section{Propensão para a Aprendizagem de Adolescentes Infratores da Lei: Reflexões a partir da abordagem biográfica}

Resumo: O estudo tem como objetivo revelar a propensão para aprender de quatro delinquentes juvenis derivados para o Programa de Salidas Alternativas da Región de Los Ríos. Assim, estávamos interessados em caracterizar o aprendizado que eles construíram, os padrões de sua propensão para aprender e os contextos em que ele se manifesta, para encontrar alternativas de modificabilidade cognitiva para apoiar as estratégias de reintegração social e educacional. Metodologicamente usamos uma abordagem interpretativa - qualitativa, utilizando a análise de conteúdo para a interpretação dos dados, seguindo a lógica da teoria fundamentada ( Strauss e Corbin, 2002) e as Histórias de Vida ( Pujadas, 1992, Bolívar e outros, 2001). Em resumo, verificou-se que a propensão a aprender com esses adolescentes é caracterizada pela repetição, a autonomia, a alta impressionabilidade e esforço bem implantado em contextos de aprendizagem informal; enfrentando períodos de ossificação progressiva dentro da escola, restringindo os seus recursos e potencial de desenvolvimento.

Palavras-chave: Propensão para Aprender, Adolescente Infrator da Lei, Aprendizagem, Modificabilidade Cognitiva, Histórias de Vida .

$* * *$

\section{Introducción}

El enfoque socio-histórico propuesto en este trabajo, exige comprender los procesos psicológicos del ser humano (el aprendizaje por ejemplo), como la interacción permanente y dinámica del sujeto con el contexto (tanto histórico como cultural), abandonando aquellas corrientes deterministas y fragmentarias del desarrollo humano. Así, la temporalidad adquiere especial importancia en las trayectorias vitales de estos adolescentes, ya que permite acceder a los diversos puntos de encuentros y desencuentros vinculados al desarrollo de su propensión a aprender ${ }^{2}$, develando la compleja red de relaciones (testigos de vida como: padres, amigos, profesores, etc.) y contextos que le permiten o no, desplegar sus recursos cognitivos desde su nacimiento hasta la época actual, descubriendo y co-construyendo una visión más profunda y enriquecida de sus propias vidas.

Por otra parte, la investigación se sitúa en la intervención de los Programas de Salidas Alternativas ${ }^{3}$ dependientes del Servicio Nacional de Menores (SENAME) los cuales tienen la misión de atender y brindar un proceso de intervención socioeducativa con todos aquellos adolescentes derivados desde los Tribunales de Justicia por su vinculación a uno o más episodios de infracción de ley, contemplados dentro de la Ley de Responsabilidad Penal Adolescente $\mathrm{N}^{\circ} 20.084^{4}$. En lo particular, nos centramos en la profundización de las Historias de Vida de 4 jóvenes que se encontraban siendo atendidos dentro del programa existente en la Región de Los Ríos, para develar y comprender como se manifiesta su propensión a aprender a lo largo de sus trayectorias vitales, las relaciones que establecen (por ejem- 
plo: a nivel familiar, grupo de pares, etc.) y de qué forma se vinculan o no a la infracción de ley, los contextos o espacios en los cuales la despliegan, para lograr descubrir los patrones que caracterizarían esta propensión al aprendizaje, y por otra parte, descubrir alternativas de reinserción social y educativa acorde a sus intereses y recursos personales.

El presente trabajo se delimita en el tiempo, a la intervención que desarrolla el investigador, como Encargado de Casos, dentro del Programa de Salidas Alternativas de la ACJ durante Junio 2011 - Diciembre 2012, con jóvenes que inicialmente eran atendidos dentro de este programa y que posteriormente fueron egresando del mismo, tras haber cumplido con la medida judicial emanada por los Tribunales de Justicia.

A nivel gubernamental, se han generado múltiples acciones respecto a la intervención con los adolescentes infractores de ley y la prevención de la delincuencia juvenil, sin conocer realmente el fenómeno ni al sujeto involucrado, desde perspectivas reduccionistas y fragmentarias. Sumado a lo anterior, el sistema educativo actual, se convierte muchas veces en un espacio inhóspito y expulsivo para estos adolescentes, al no tomar en cuenta sus recursos y experiencias previas, estableciendo indirectamente, procesos de marginación y exclusión que terminan por aumentar las tasas de fracaso y deserción escolar. De tal modo, que el problema central de esta investigación, radicó en poder esclarecer: ¿Por qué un adolescente infractor de ley aprende con tanta facilidad en contextos y espacios informales?, ¿Por qué se le dificulta tanto aprender dentro del sistema escolar formal (escuelas, liceos, etc.)?, y ¿qué es lo que ocurre con la propensión a aprender de estos adolescentes a lo largo de sus Historias de Vida?

La investigación se posiciona dentro del paradigma interpretativo cualitativo, que explora descriptiva e interpretativamente, cómo se manifiesta la propensión a aprender en los adolescentes infractores de ley. Dicha realidad ha sido escasamente investigada por las ciencias sociales y de la educación, razón por la cual, la investigación es de carácter exploratorio al observar el fenómeno en estudio tal y cómo se da en su contexto real y cotidiano lo que refleja, claramente, el carácter no experimental de nuestro trabajo. Metodológicamente utilizamos un enfoque interpretativo - cualitativo, recurriendo al análisis de contenido para la interpretación de los datos, siguiendo la lógica de la Teoría Fundamentada (Strauss y Corbin, 2002) y las Historias de Vida (Pujadas, 1992; Bolívar y Otros, 2001). El artículo pretende concluir con los principales hallazgos que emergieron del estudio, además de una serie de recomendaciones extraídas del mismo, para mejorar los procesos de intervención socioeducativos que se desarrollan en el trabajo con los adolescentes infractores de ley en Chile.

\section{Formulación del Problema}

En la actualidad, el sistema educativo formal rechaza e invalida la experiencia previa con la que cuentan los adolescentes vinculados a un 
episodio de infracción de ley, dado que la escuela "ha desarrollado un proceso implacable de apropiación y monopolización ilegitima de la educación, imponiendo un modo de ser escolar excluyente de otros modos posibles” (Calvo, 2008: 237). De esta forma, todo aquel repertorio de experiencias previas que desarrolla un adolescente, a lo largo de su vida, es desechado por el sistema educativo formal, ya que no se acopla a la planificación, programas y objetivos institucionalmente instaurados como parte del proceso de enseñanza de todo adolescente. Ante este panorama tan adverso para el adolescente infractor de ley y su vinculación a la educación formal, se produce un quiebre y un desencantamiento significativo, dado que no se le permite desplegar adecuadamente sus potencialidades y recursos personales, ahogándolo en un marco normativo intenso y rígido, que termina por precipitar el fracaso y la deserción escolar, "proceso que, en gran parte de los casos, ha sido acompañado por repitencias, bajos rendimientos, abandonos parciales, expulsiones, entre otros" (Flamey, 2006: 5). Es posible ver con qué facilidad aprende un adolescente en el contexto informal, descubre y establece nuevas relaciones posibles. De manera que "la lista de experiencias y aprendizajes es infinita en su diversidad, pero todas tienen en común el haber sido emergentes, caóticas, aleatorias y profundas en nuestra vida" (Calvo, 2010: 93).

Sin embargo, esta enorme propensión a aprender que posee un adolescente vinculado a uno o más episodios de infracción de ley, se ve absolutamente paralizada y coartada dentro del sistema escolar, donde manifiesta una gran dificultad para aprender en contextos tan normativos. De tal modo, que lo interesante y valioso de esta investigación, giró en torno a poder descubrir y explorar qué es lo que acontece con la propensión a aprender del adolescente infractor de ley a lo largo de su trayectoria vital, los cambios que sufre y en qué contextos adquiere mayor desarrollo.

Acorde a lo anterior, resultó interesante comprender y caracterizar la complejidad que encierra un acto delictivo (las habilidades, recursos y potencialidades que utiliza un adolescente en esta situación, bajo contextos informales de la vida cotidiana), y por qué no despliegan todos estos recursos en el contexto escolar (educación formal, ambiente de trabajo normativo, estructurado y lineal), pero sí en el ámbito de la delincuencia juvenil, vinculado al grupo de pares, pandillas y subculturas juveniles, con los cuales comparte valores, intereses, costumbres y trayectorias vitales similares.

\section{Marco Teórico - Conceptual Educación y aprendizaje informal}

En la educación informal, que sucede fuera de la escuela, en espacios y tiempos también informales "representa los procesos educativos, que tienen lugar en la calle, el café o el patio escolar" (Calvo, 2002: 228), donde la ocurrencia del proceso de enseñanza y de aprendizaje se encuentran unidos complementariamente, uno en relación con el otro, 
dialógicamente. En palabras de Moreno y Calvo (2010: 134), sujetos a "las emergencias que bifurcan los procesos de manera aleatoria, donde cada uno sigue el proceso a su ritmo y estilo, si bien la permisividad no es total, es más abierta que la escolar”.

El aprendizaje informal es polifacético, ya que no se reduce a uno solo por vez, sino a varios simultáneamente que emergen continuamente. "La simultaneidad se relaciona con la sinergia y el carácter transdisciplinario del saber” (Calvo, 2002: 231). Por ende, la potencialidad de la educación informal radica en permitir que el alumno, durante el proceso de aprendizaje fluya caóticamente, ya que este fluir genera y permite el despliegue de la creatividad y la curiosidad por aprender, el surgimiento de preguntas novedosas que le permitan descubrir el mundo, pero que en el sistema formal, obligarían al profesorado a imponer esa línea causal del control y el poder que le otorga ser el poseedor del conocimiento existente en el aula.

El aprendiz en la educación informal es quien determina lo que quiere aprender (qué), el tiempo en que quiere aprender (cuándo) y hasta cómo lo quiere aprender (Rogers, 2004; en Gómez, 2009: 40). Es la acción difusa y no planificada que ejercen las influencias ambientales, tales como la familia, el trabajo, el grupo de pares, la comunidad, los medios de comunicación, etc. Por lo tanto, un niño en situaciones educativas informales se esfuerza por aprender más que en la escuela, pero nunca más allá de lo que le es posible según su maduración y desarrollo cognitivo, ni por debajo de sus capacidades. (Calvo, 2002: 234).

\section{Experiencia aprendizaje mediado}

Bajo esta perspectiva, el desarrollo cognitivo de los individuos no dependería exclusivamente de la capacidad que éstos tengan de obtener beneficios de los estímulos y aprendizajes extraídos de su entorno cercano, sino que podría ser potenciado a través de experiencias de aprendizaje mediado, donde un tercero (en tanto mediador), es quién acerca el mundo al sujeto, organizando y seleccionando aquellos estímulos más relevantes del entorno, para que el propio sujeto, interactúe con ellos y despliegue al máximo sus recursos cognitivos, logrando la adquisición de aprendizajes relevantes y novedosos para su adaptación al medio. Así, es necesario recurrir a los aportes de Feuerstein y Otros (1998), quien define la experiencia de aprendizaje mediado, como toda “interacción durante la cual el organismo humano es objeto de la intervención de un mediador. El aprendiz, a través de esta interacción, se forjan en él, un repertorio de disposiciones, propensiones, orientaciones, actitudes y técnicas que le permitan modificarse con respecto a otros estímulos". De tal forma que el desarrollo cognitivo que un sujeto podría lograr a lo largo de su trayectoria vital, estaría dado por la capacidad de cambio, flexibilidad y adaptabilidad que tenga para avanzar desde un nivel de funcionamiento, hacia otro más complejo y elevado que le permita enfrentar y resolver nuevas situaciones o problemas. 
De modo que esta modificabilidad cognitiva, fluye en el organismo de manera impredecible y compleja desde el nacimiento, como parte de su propensión y adaptabilidad al cambio. Justamente, esta serie de cambios dejarían una huella significativa y profunda en los sujetos, al tener impacto directo en su estructura del pensamiento, obligándolo a reestructurarlo y modificarlo. Al respecto, López de Maturana (2010: 45 -46), señala que la modificabilidad cognitiva sería "un fenómeno humano, que a través de condiciones externas como la Experiencia de Aprendizaje Mediado o las necesidades determinadas por una situación particular, provocan en los sujetos conductas que previamente no existían en su repertorio”.

Es preciso recalcar que la experiencia de aprendizaje mediado y la posibilidad de modificabilidad cognitiva en los individuos, sólo sería posible alcanzarla, de acuerdo con Feuerstein (1983), a través del establecimiento de ambientes activos modificantes, es decir, aquellos contextos y situaciones que generan calidades e intensidades de interacción activas y profundas entre el individuo y un otro (mediador), el cual invita al individuo a situaciones desafiantes, a la solución de problemas complejos y heterogéneos, que actuarían como productores y amplificadores de cambio en las estructuras del pensamiento, y por ende, de experiencias de aprendizaje que impulsan el desarrollo cognitivo del sujeto y de su verdadero potencial de aprendizaje.

Sería posible inferir entonces que la mayor tasa de fracaso y deserción escolar que experimentarían los adolescentes infractores de ley, sería producto de los determinantes distales que lo afectan y no por su incapacidad para aprender. De tal forma que si estos adolescentes se desarrollan cognitivamente en un medio con determinantes distales ${ }^{5}$ (caracterizados por una alta deprivación sociocultural, disfuncionalidad familiar, carencias socioeconómicas, bajo nivel de estimulación por parte de los padres, etc.) y un bajo impacto de los determinantes proximales, el panorama escolar podría ser bastante sombrío. Afortunadamente, aquí cobran sentido los aportes de Feuerstein (1991), ya que todos estos factores podrían ser revertidos por medio de los determinantes proximales del aprendizaje, que movilicen al adolescente hacia experiencias positivas de aprendizaje mediado, donde pueda identificar y desplegar al máximo sus potencialidades y recursos cognitivos.

\section{Escuela y aprendizaje formal}

La educación formal limita las oportunidades de desarrollo de un joven, en tanto el sistema escolar transmite que estudiar es difícil, que el juego está proscrito mientras se aprende y que el fracaso escolar sucede porque el alumno no se dedica lo suficiente, coartando gradualmente sus verdaderas potencialidades y recursos. Bajo este marco dominante de la educación formal, estructurada y rígida, es desde donde "la escuela se refugia en el carácter normativo y profetiza del fracaso escolar" 
(Calvo y Elizalde, 2010: 9). Desde esta óptica lineal, que privilegia el orden, por sobre el caos, es que surgen múltiples formas de castigos y sanciones represoras hacia quiénes se alejen de lo formalmente establecido (procesos de escolarización), afectando drásticamente a los alumnos principalmente, e incluso a los profesores y directivos, si se alejan de la estructura predominante. Todo esto, hace que se acreciente la negativa del joven por enjaular su educación en la escuela (escolarización), y ante la evidente dificultad en aceptar normas y reglamentos internos de una determinada unidad educativa, se produce una desconexión entre el joven y el sistema escolar, dado que no satisface sus expectativas y prioridades personales (INJUV, 2007; 2009: 48).

Así, se produce un quiebre y un rechazo hacia los representantes del sistema escolar que desconocen las potencialidades y recursos individuales de los adolescentes, su creatividad e imaginación; al cuestionar recurrentemente su bagaje socio-cultural, catalogándolos como un problema (dada su condición de adolescente infractor de ley), viendo entre sus posibilidades, la deserción escolar ante un sistema, que en vez de acoger, contener y apoyar, restringe, limita, niega y "domestica las potencialidades del joven, de manera que su rol en la escuela y frente a sus profesores, es pasivo y más bien desafectado de lo que allí sucede" (Muñoz, 2008: 67). Según González (1996: 55 - 61), esto ocurre tras "la marginación de la sociedad por su forma de vestir, hablar, o comportarse; por la utilización de la violencia para defenderse de otros grupos; impulsividad; autarquía”, como un intento de resolver sus problemas por sus propios medios, "sin contar con los adultos (familia); evasión y mentalidad mítica, manifiesta en tabúes, ritos, tatuajes, etc.”. Ciertamente, el grupo de pares se convierte en fuente de apoyo, comprensión, afecto, confianza y otros elementos que los cohesionan, no como elementos aislados y a la deriva, sino como grupo con intereses y características similares. Tristemente, el joven infractor de ley se ve enfrentado a la cruda realidad donde el desarrollo de la desviación arrancaría en la escuela, desplazándose posteriormente a la calle (grupo de pares) y a ciertos ámbitos del barrio, nutriéndose de otras prácticas y articulaciones simbólicas juveniles (Tsukame, 1996: 2010: 44).

Dado que la mayor parte de los estudios e investigaciones educativas, en la actualidad, están centrados en el aprendizaje que ocurre en la educación formal (Gómez, 2009: 39), sumado a que el actual modelo de justicia juvenil restaurativo existente en Chile, dialoga inarmónicamente con el aprendizaje y la verdadera potencialidad de estos jóvenes. El desafío está en poder abordar, a través de esta investigación, cómo se despliega la propensión a aprender en los adolescentes infractores de ley, el medio informal en el que aprenden estos adolescentes y sus diversas formas, para apoyar procesos de integración social efectivos a través de los procesos educativos que tienen lugar dentro y fuera de la escuela, que permitan disminuir la reinserción penal del adolescente. 


\section{Sistemas de Justicia Juvenil: Una Mirada desde la Educación}

Al explorar y contrastar los diversos modelos de justicia juvenil existentes en América Latina, que surgen a partir de mediados de la década del '90 y “como consecuencia, en gran medida, de la aprobación de la Convención Internacional sobre los Derechos del Niño” (Beloff, 2004: 1), es posible apreciar que existe un gran desarrollo y transformación en términos jurídicos, que incorporan la visión del adolescente como un sujeto de derecho, con mayores libertades y con clara conciencia de sus actos, por ende, existe consenso en cuanto a la edad de imputabilidad (en promedio, desde los 14 hasta los 18 años), las penas que se le apliquen cuando cometa una infracción de ley (penas privativas y no privativas de libertad, aplicación de medidas socioeducativas, acuerdos reparatorios, soluciones alternativas al conflicto etc.) y que giran en torno a la responsabilización del adolescente frente al delito.

No obstante, carecen en su mayoría de mecanismos institucionales que permitan conectar al adolescente vinculado a uno (o varios) episodios de infracción de ley con la educación, lo que en definitiva hace que se convierta en un medio institucionalizado "para la exclusión y marginación social” (Mendicoa y Otros, 1999:53-54). Junto a ello, se observa un escaso grado de articulación entre las políticas públicas más relevantes para el adolescente, donde no existe un diálogo institucional (Justicia, Educación, Trabajo, Cultura, etc.), que permita verdaderamente atender a las reales necesidades de un joven que se involucra en una situación de infracción de ley, sino tan sólo, existe interés en la aplicación de medidas correctivas de dicho comportamiento, para disminuir la posibilidad de que desarrolle una trayectoria delictiva vital, "el reconocimiento de las garantías adolescentes, la reducción del ámbito de lo penal o a su abolición” (Beloff, 2004: 38).

En la actualidad, la lógica epistemológica cartesiana del proceso escolarizante (Calvo, 2008: 89), se caracteriza por la entrega de habilidades y conocimientos (de manera planificada y secuencial), la corrección disciplinaria de todos aquellos comportamientos indeseados (que perturben y dificulten la transmisión mecánica y asimétrica de la enseñanza) y la inserción en el mundo externo (al entorno familiar), dado que la escuela ha sido y continúa siéndolo, un espacio eminentemente normativo, que transmite códigos muy diferentes a los que posee un adolescente infractor de ley, y que además, no permite que el azar, la creatividad, la autonomía y la improvisación cumplan su rol educativo, por ende, no permite una relación dialógica con el sistema de justicia juvenil actual existente en América Latina. Ante este panorama tan adverso para un adolescente infractor de ley, el adaptarse a la escuela se transforma en un foco de tensión y conflicto constante, y por otra parte, la escuela no logra adecuarse a las características diversas de este tipo de jóvenes, precipitándolo de alguna forma, a la deserción escolar, la cual "mientras más precoz, genera más posibilidades de que el adolescente presente conductas delictuales" (Gottfredson y Otros, 1996; Fernández, 2003: 5). 
En la mayoría de los casos, la escuela (a través de la enseñanza), superficializa, reduce y complica los procesos, mientras que la educación los simplifica y complejiza. "La diferencia reside en contar o no con criterios orientadores” (Calvo y Elizalde, 2010: 8). El fracaso escolar tendría su origen en la concepción de enseñanza y de aprendizaje que sustenta a la escuela (concebido de manera secuencial, lógica, lineal) y no en el alumno, profesor, familia o grupo de pares, ni en la adecuación o pertinencia de los planes y programas de estudio.

El cambio educacional que requerimos no pasa por la implementación de una Reforma formal de la escuela, los planes y programas de estudio y las formas de evaluar. Por el contrario, debemos discontinuar el trabajo escolar desescolarizando a la escuela" (Calvo, 2008; Calvo y Elizalde, 2010: 7). Resulta fundamental reconocer la inmensa potencialidad que encierra la educación informal, dado que es universal y permanente, y llegar a comprender que "todos nos educamos en todo momento" (Ibid, 2008: 240).

De tal manera que en muchas ocasiones, la escuela de manera implícita o explícita niega, coarta y reprime las experiencias previas de los adolescentes infractores de ley, desconociendo la facilidad y destreza con que éstos aprenden en otros espacios (fuera de la escuela), desplegando aprendizajes extraordinarios y complejos, como por ejemplo: liderazgo y autonomía para tomar decisiones colectivamente (al interior del grupo de pares, donde se observan jerarquías y dinámicas organizacionales complejas), adquisición de un pensamiento pragmático abstracto (para enfrentar el día a día, en un medio hostil), resiliencia y tolerancia a la frustración (para enfrentar marginación y exclusión social, la falta de oportunidades), afición por la música (desarrollo del arte callejero y todo tipo de tribus urbanas como punk, góticos, skatters, donde plantean críticas a la sociedad), etc.

\section{Diseño metodológico}

La presente investigación la desarrollamos desde un enfoque interpretativo cualitativo, ya que "es un proceso interpretativo de indagación basado en distintas tradiciones metodológicas, entre ellas, la biografía, la fenomenología, la teoría fundamentada en los datos, que examina un problema humano o social. De este modo, nos apoyamos en 2 tradiciones teóricas relevantes para la investigación social y educativa. Una primera tradición, corresponde a la Teoría Fundada (Strauss y Corbin, 2002). La segunda corresponde a las Historias de Vida (Pineaud y Jobert, 1989; Pineau y Le Grand, 1993; Bolívar y Otros, 2001).

Así, la Teoría Fundada, se convierte en una teoría derivada de datos recopilados de manera sistemática y analizados por medio de un proceso de investigación, donde la "recolección de datos, análisis y la teoría que surgirá de ellos guardan estrecha relación entre sí. Un investigador no inicia un proyecto con una teoría preconcebida, sino más bien comienza con un área de estudio y permite que la teoría emerja a partir de los datos" (Strauss y 
Corbin, 2002: 13 - 14). De tal manera que la teoría derivada de los datos tendrá mayor grado de cercanía y profundidad con la realidad en estudio (a través del método de la comparación constante), aumentando la posibilidad de que permita la generación de nuevos conocimientos, aumenten la comprensión y proporcionen una guía significativa para el ámbito en estudio. Asimismo, se convierte en un recurso metodológico flexible y dinámico que permite, según Vasilachis (2006: 171), lograr un mayor grado de profundización conceptual sobre las temáticas centrales de la investigación (en este caso, develar y comprender la propensión a aprender de los adolescentes infractores de ley) y por otra parte, permite de manera complementaria y dependiendo los intereses del investigador (número de participantes en la investigación, cantidad y calidad en la información que se obtendrá, plazos para la recolección e interpretación de la información, etc.), la utilización de diversos programas informáticos cualitativos (como por ejemplo, el uso del Atlas Ti), que potenciarían la posibilidad de producir investigación cualitativa con mayor rigor intelectual.

Del mismo modo, las Historias de Vida de relatos paralelos (Pujadas, 1992: 14), nos permitieron analizar e interpretar los hechos de la vida de una persona, para comprenderla en su singularidad o como parte de un grupo, "de su experiencia de largo plazo, contada a un investigador y/o surgida del trabajo con documentos y otros registros vitales” (Vasilachis, 2006: 176). Además, y de acuerdo con Miller (2000: 02), las historias de vida tienen dos implicancias centrales para la investigación cualitativa: la primera es la centralidad que adquiere el tiempo en la historia de vida, "más que otros abordajes centrados en el presente, este enfoque construye su práctica en la relación entre pasado, presente y futuro que expresa el entrevistado. $\mathrm{La}$ segunda, es la importancia de la familia (la de origen y la formada por el entrevistado), en la vida de las personas, rompiendo con la ficción de los individuos atomizados”. De tal forma, esta perspectiva de análisis nos posibilitó descubrir y explorar lo cotidiano, las prácticas de vida de los adolescentes vinculados a un episodio de infracción de ley, las principales problemáticas que lo afectan, tomando en cuenta y validando lo que ocurre en el día a día, nos permite conocer desde dentro lo que ocurre en el mundo del adolescente. Para ello, utilizamos la Historia de Vida, basada en las entrevistas en profundidad semi-estructuradas (Flick, 2004), la cual permite conocer y develar, íntimamente a las personas, objetivar lo subjetivo y mirar el mundo desde sus propios ojos, como herramientas cualitativas para la obtención de datos. El trabajo de campo se desarrolló en 3 instancias: construcción de líneas de tiempo, autobiografías y entrevistas en profundidad. A partir de la información recopilada en las primeras fases del trabajo de campo (en la construcción de las Líneas de Vida y las Autobiografías aportadas por los participantes), y tras realizar el proceso de codificación y transformación de los datos, se procedió a la elaboración de las temáticas y preguntas orientadoras que conformarían el guión de las entrevistas en profundidad, que la presente investigación definió para lograr develar y comprender la propensión a aprender de los adolescentes infractores de ley. De tal modo, que cada una de estas preguntas nos permitieron enriquecer los alcan- 
ces y proyecciones de este estudio, para lograr cumplir con los objetivos planteados al inicio de la investigación.

El proceso de codificación, reducción de los datos y categorización, se realizó con el apoyo de la herramienta informática Atlas-Tí ${ }^{6}$, que nos facilitará la organización de la información para la posterior codificación, reducción y categorización de los datos. Es así como una de las grandes ventajas de incluir estas herramientas informáticas, en la investigación cualitativa, son la velocidad en el manejo de los datos (Seale, 1999), la gestión, búsqueda y exposición de los datos, la potencialidad y multiplicidad de relaciones que se pueden establecer, que facilita un mayor volumen y aprovechamiento de los datos e información recopilada durante la investigación. Otra ventaja de la utilización de herramientas informáticas, en la investigación cualitativa, es que permite un aumento de coherencia en los procedimientos analíticos (Weitzman, 2000), sumado a que logra brindar un marco de transparencia durante el proceso investigativo, facilitando la reflexión permanente, el almacenamiento único y ordenado de la información en un computador (notas de campo, observaciones, exposiciones, etc.), que brinde un marco óptimo para la generación de conocimiento.

\section{Resultados obtenidos}

De acuerdo a lo anterior, el trabajo de campo y posterior estudio de los datos permitió el levantamiento de las siguientes categorías de análisis o temas ejes, emanados a partir de las Historias de Vida de los cuatro Adolescentes Infractores de Ley que conformaron este estudio:

- Patrones de la Propensión a Aprender.

- Factores Posibilitadores de Modificabilidad Cognitiva.

- Deseos de Superación Personal.

- Configuración de Resiliencia de Adolescentes.

- Contextos y Situaciones de Aprendizaje.

- Aprendizajes Desplegados por Adolescentes.

- Trayectorias Escolares.

En cuanto a los Patrones de la Propensión a Aprender que poseen estos adolescentes, esta se caracteriza por: la repetición (como mecanismo natural de aprendizaje), el esfuerzo y la perseverancia, la autonomía y libertad, una alta influenciabilidad e impulsividad (Andrews y Bonta, 2006), donde los adolescentes dirigen sus recursos cognitivos, de manera autoorganizada hacia actividades que les parecen relevantes, novedosas y atractivas; sin mediar en las consecuencias de tales acciones, explicándose de alguna forma los episodios de infracción de ley, judicializados o no, que han cometido estos adolescentes a lo largo de sus trayectorias vitales. Asimismo, se desarrolla entre procesos permanentes de observación, imitación (inédita y subjetiva) y emulación de miembros específicos de la sociedad (Calvo, 2008: 65), puesto que la adolescencia es una etapa evolutiva caracterizada por la vinculación de los jóvenes al grupo de pares, donde 
comienzan a desarrollar y adquirir nuevos y diversos aprendizajes, los cuales muchas veces no son reconocidos o valorados adecuadamente dentro de espacios formales de aprendizaje (representado por los liceos a los que pertenecen), sin embargo, son relaciones que permiten comenzar a configurar su identidad (como meta evolutiva significativa para un adecuado desarrollo humano). En este sentido, resulta interesante observar como la influenciabilidad que caracteriza la propensión a aprender de estos adolescentes no siempre es negativa (al proporcionar a los jóvenes una identidad provisional que pueden adoptar, por lo menos temporalmente, hasta que alcancen una identidad más estable en fases posteriores del desarrollo), ni uniforme (siendo capaces de alternar su inserción en diversos grupos de pares, como también en otros espacios comunitarios, tales como la Iglesia, el deporte, el mundo laboral), ampliando su repertorio de aprendizajes y potenciando diversas habilidades o recursos (que muchas veces desconocían).

Asimismo, es posible identificar que la propensión a aprender de estos adolescentes alcanzaría un desarrollo óptimo en contextos de aprendizajes abiertos, contextualizados y donde los propios jóvenes puedan convertirse en los protagonistas de su proceso de aprendizaje, reconociendo el carácter social que éste tiene y fomentando el deseo de aprender en diversos espacios y contextos, validando cada una de sus experiencias previas. Estas condiciones y oportunidades de desarrollo experimentarían cierto nivel de estancamiento / anquilosamiento dentro de contextos formales de aprendizaje, espacios en los cuales la norma o regla, se convierte en la guía ordenadora de los procesos de desarrollo de los adolescentes, propiciando una fuerte tendencia homogeneizadora que artificializa y limita la propia experiencia del aprender, siendo que esta es precisamente una cualidad humana innata, pero que al escolarizarse, las intenciones educativas no trascienden más allá del mero discurso.

En relación a los Factores que posibilitarían la Modificabilidad Cognitiva en estos adolescentes, estaría influenciada por la existencia de los siguientes elementos:

- Altos niveles de resiliencia desplegados a lo largo de sus trayectorias vitales.

- Relaciones Afectivas Significativas: los cuales de una u otra forma, adoptan características básicas de un mediador en potencia (tal como un profesor, amigo, padre, hermano, etc.), capaz de identificar y potenciar sus recursos y potencialidades; teniendo una visión positiva de ellos (evitando la estigmatización), transmitirle confianza y afecto, reflejando, en parte, algunos de los criterios básicos que posibilitan las Experiencias de Aprendizaje Mediado, tales como la intencionalidad y reciprocidad propuestos por Feuerstein (1991), al compartir las intenciones con el sujeto, en un proceso mutuo de enriquecimiento y desarrollo, que valore las capacidades y potencialidades de estos adolescentes. 
- Inserción Comunitaria Significativa: espacios y contextos en los cuales los adolescentes reciben apoyo y orientación en la superación de aquellas experiencias de fracaso (tal como una repitencia escolar, vinculación a un episodio de infracción de ley, etc.).

- Importancia del Compartir (Moreira, 1997): entendiéndolo como una actividad permanente, significativa y relevante para el adolescente, haciéndose necesario que los estímulos que se le presenten, se tornen atractivos e interesantes (de manera gradual). Por lo tanto, es imprescindible que la información y/o contenidos (que se pretenden mediar con los jóvenes) sea reveladora y altamente significativa, desde el punto de vista de su estructura externa, para que sea coherente con su realidad; clara, organizada y factible de ser relacionada con sus esquemas de conocimiento previos existentes en su estructura cognitiva.

- Deseos de Superación Personal, por medio de la Definición de un Proyecto de Vida en los adolescentes, siendo capaces de fijarse metas desafiantes y complejas que le permitan, por un lado, desenmascarar su verdadero potencial de aprendizaje y al mismo tiempo, favorecer sus propios intereses.

Con respecto a los Contextos y Situaciones de Aprendizaje, los jóvenes se despliegan desde lo formal a lo informal, alcanzando una amplia gama de aprendizajes, habilidades y destrezas, haciendo posible inferir, que la propensión a aprender que estos poseen se manifiesta con mayor claridad en contextos informales de aprendizaje (multicancha, sector agrícola, plaza de juegos, espacios públicos, etc.), fluyendo de manera autoorganizada, posibilitando la adquisición de una amplia gama de destrezas y habilidades (muchas veces desconocidas hasta por los propios jóvenes), reflejando un amplio repertorio de funciones cognitivas cuando se analiza minuciosamente un acto delictivo (tales como la toma de decisiones, abstracción y conceptualización, planificación, observación, comparación, razonamiento, memorización, atención, reorganización, etc.), con un alto valor educativo.

No obstante a lo anterior, esta enorme propensión al aprendizaje experimenta periodos de profundo anquilosamiento cuando los jóvenes ingresan al sistema escolar formal, limitándose dramáticamente sus verdaderas potencialidades y recursos, cuando ingresan en la etapa de la adolescencia y de manera autónoma desafían permanentemente las normas y tendencia homogeneizadora de la escuela, optando muchas veces por el abandono escolar.

Estos adolescentes, según Hein (2004), logran ser bastante hábiles obteniendo el máximo beneficio de su entorno, tienen una marcada tendencia a distraerse con facilidad, a mostrar extrema confianza en sí mismo y pereza en el entrenamiento del pensamiento crítico (basado en la lógica, la precisión y la exactitud, perspectiva predominante en el espacio escolar 
formal. Sin embargo, esto no quiere decir que los adolescentes no puedan desarrollarlo, sino que simplemente no les interesa hacerlo, dado que no es requerido en los contextos en los cuales se desenvuelven). También se ha observado que tienden a estar orientados hacia el poder (se definen y validan frente a los pares, por medio de la fuerza y destreza física), junto con interpretar el mundo como un espacio tremendamente hostil. Esto explicaría el por qué los adolescentes vinculados a episodios de infracción de ley revelan (intencionalmente, la mayoría de las veces), un menor desarrollo de habilidades sociales (como la empatía o la asertividad), dado que a lo largo de su trayectoria vital han aprendido (con mayor o peor suerte), a sobrevivir y superar las adversidades que obstaculizan su desarrollo.

En lo que respecta a las trayectorias escolares, descritas como aquel conjunto de experiencias, percepciones y vivencias identificadas por los adolescentes, durante su paso o permanencia dentro del sistema escolar formal (representado por los procesos de escolarización tradicionales existentes en Chile, tales como: jardín infantil, escuela básica, liceos de enseñanza media, etc.), los cuales han contribuido a la generación de una serie de creencias negativas y de bajo impacto en su aprendizaje. Entre otras cosas, debido a que están marcadas por una alta rotación escolar puesto que exhiben una baja asistencia, comportamiento disruptivo en el aula y marcada tendencia hacia la deserción escolar, razón por la cual son catalogados como estudiantes problemáticos que no se acomodan a los Proyectos Educativos Institucionales. Esta situación se acrecienta principalmente durante la enseñanza media, donde los adolescentes comienzan a interiorizar un desencantamiento y decidido rechazo hacia los representantes del espacio escolar, quienes muchas veces descartan las potencialidades y recursos individuales de los adolescentes, su creatividad e imaginación; al cuestionar recurrentemente su bagaje socio-cultural y condición de infractor de ley.

Por consiguiente, estos jóvenes están más expuestos a vivenciar trayectorias vitales marcadas por transiciones rotas (Willis, 1988), en el camino por asumir roles adultos, es decir, experiencias escolares de fracaso y modelos de intervención individual paliativos, pero no educativos. Es así como en palabras de Foucault (1996), el sistema educativo actual no estaría cumpliendo su función igualitaria, al contrario, éste ha actuado y continua promoviendo funciones de socialización de una cultura homogénea, que opera seleccionando y distribuyendo recursos humanos de forma clásica para la mantención del status quo y la perpetuación de la desigualdad social imperante que enriquece a unos pocos, en desmedro de muchos.

Sin embargo, no todos los aprendizajes que adquieren estos jóvenes durante su permanencia en el sistema escolar son negativos, sino que también les permite incorporar ciertos valores esenciales para la vida (tales como la responsabilidad, tolerancia, respeto, esfuerzo, honestidad mencionados por la totalidad de los participantes en este estudio), convirtiéndose en un agente socializador, que muchas veces, supera y fortalece las experiencias previas de estos adolescentes, al provenir de contextos familiares disfuncionales, entornos deprivados socioculturalmente. Esta transmisión 
de valores concuerda con otras investigaciones en las cuales se revela que el sistema escolar formal influye en todos los aspectos relativos a los procesos de socialización e individuación del adolescente, entre los que destacan: "el desarrollo de las relaciones afectivas, la habilidad para participar en situaciones sociales, la adquisición de destrezas relacionadas con la competencia comunicativa, el desarrollo del rol sexual, el desarrollo de las conductas prosociales y el desarrollo de la propia identidad personal (Montero, 2000).

Otro de los factores relevantes observados en la trayectoria escolar de estos adolescentes, radica en el profundo distanciamiento que existe entre las familias de estos jóvenes y el sistema escolar formal al que pertenecen, debido a que las instancias de diálogo y colaboración planificadas desde la escuela (tales como reuniones de apoderados, actividades recreativas, orientación escolar, etc.) son desaprovechadas por estas familias, acercándose sólo ante situaciones particulares donde los adolescentes son definidos como un "problema” para el establecimiento educativo debido a que no logra adaptarse a las normativas vigentes dentro de este contexto. Por otra parte, los adolescentes reconocen y valoran negativamente la calidad de la infraestructura de sus establecimientos educacionales (debido entre otras cosas a que las aulas carecen de una adecuada calefacción y ventilación, deterioro significativo de puertas, mobiliario y ventanas, bajo nivel de satisfacción frente a las raciones alimentarias, precaria presencia de material recreativo, tales como mesas de pin pon, etc.). Al respecto Lombaert (1999), sostiene que los adolescentes infractores de ley fracasan en la escuela porque muchas veces el establecimiento escolar les ofrece un paquete no adecuado (desconociendo sus experiencias previas e Historias de Vida); desarrollan un autoimagen y perspectiva social inadecuada, porque el colegio no da para más; por eso buscan una autovaloración alternativa en grupos no-conformistas (de ahí la propensión a relacionarse con grupo de pares con los cuales comparten intereses, valores y experiencias similares asociadas a contextos de origen vulnerados); y por ende, son frágiles ante el mercado laboral y mal ubicados en la escala social imperante dentro de un país como Chile, donde el sistema Neoliberal alcanza un gran progreso económico, en desmedro de una mayor desigualdad social. De tal modo que muchos de estos adolescentes, entran en conflicto con las instituciones tradicionalmente reconocidas como entes socializadores por excelencia (tales como la familia, escuela, etc.), producto de un cúmulo significativo de experiencias escolares negativas, marcadas por la exclusión, rotación, repitencia, fracaso y/o deserción escolar, enfrentando reiteradamente el rechazo de esta institución, la cual potencia una profunda confrontación con este sistema, debido al desencuentro entre sus expectativas e intereses personales versus lo socialmente esperable, pugna que se resuelve imponiéndose el poder que tenga una de las partes, culpabilizando y criminalizando a la otra. En este mismo sentido, se observa en las trayectorias escolares de éstos jóvenes que el haber tenido alguna vez malos resultados escolares (desde la lógica escolar imperante que los ha evaluado), las suspensiones y faltas en la escuela, la alta rotación escolar, la dificultad para obedecer órdenes y tener discusiones algunas veces con compañeros no es un indicador de que se genere la conducta antisocial y delictiva; pero 
la acumulación de estos factores de riesgo en un adolescente, pueden interactuar y aumentar la probabilidad de que se presente el comportamiento antisocial y delictivo (Ezpeleta, 2005).

\section{A modo de conclusiones}

Organizamos estas reflexiones, a modo de conclusiones, guiándonos por los objetivos específicos que originaron el presente estudio.

En primer lugar, con respecto a los patrones que integrarían la propensión a aprender de estos adolescentes vinculados a episodios de infracción de ley en la Región de Los Ríos, podemos observar que esta se caracterizaría:

- La repetición haciendo alusión a la memorización, más que al complejo y armónico funcionamiento de la memoria que no aprovechan ni estimulan adecuadamente dentro de la escuela.

- La autonomía, constancia y esfuerzo que potencian la exploración, el descubrir, el comprender, la autogestión e independencia dentro de los diversos ámbitos en los que se desenvuelven, pero que fluye de manera caótica y flexible en espacios informales de desarrollo.

- Alta influenciabilidad en asociación al grupo de pares, propio de la adolescencia y en la cual son capaces de experimentar cambios profundos en su comportamiento, desafiando la norma y los límites impuestos por los agentes socializadores como la familia y la escuela, ampliando ostensiblemente sus experiencias y aprendizajes previos.

- La impulsividad: observándose un bajo nivel de autocontrol, manejo de la frustración y agresividad frente a aquellos espacios, contextos o situaciones en las cuales se ven restringidas sus posibilidades de desarrollo o realización, tal como ocurre dentro del sistema escolar formal donde se les anula la curiosidad natural e implantan arbitrariamente lógicas de aprendizaje homogeneizadoras.

- Cursaría Periodos de Anquilosamiento en Contextos Formales de Aprendizaje. Este anquilosamiento se explicaría porque el sistema escolar se convierte en un ambiente pasivo aceptante, donde hay ausencia de experiencias de aprendizaje mediado (no hay intencionalidad, reciprocidad, mediación del significado, etc.), reduciendo el sentido de competencia del adolescente (asociado al ser capaz de algo y no a la competitividad), invisibilizando y desaprovechando sus verdaderas potencialidades.

En segundo lugar, y en relación a los aprendizajes construidos por estos adolescentes hemos podido caracterizar y descubrir una gran diversi- 
dad y heterogeneidad de aprendizajes exhibidos por estos adolescentes. De modo tal que dentro los procesos de escolarización, han podido adquirir conocimientos teórico - prácticos (dentro de un currículum rígido y planificado, asociado a las matemáticas, lenguaje e historia por ejemplo), el respeto a las personas, el sentido de la responsabilidad (guiando su comportamiento muchas veces por temor al castigo), la importancia de construir un proyecto de vida a futuro (es decir, definiendo su futuro laboral y productivo, más que algo relacionado a sus intereses y/o verdaderas potencialidades), manejo y acceso de las tecnologías de la información (como un computador, celular, redes sociales, etc.), importancia del autoconocimiento, etc. Por otra parte, dentro de contextos no formales de aprendizaje (tales como la Iglesia, Club Deportivo de Fútbol, Movimiento Scout, etc.), los adolescentes han podido valorar y reconocer la importancia del respeto y los valores entre las personas, la importancia de la disciplina y la adaptación a las normas socialmente impuestas (leyes, mandatos parentales, etc.), desarrollo habilidades musicales (tocando un instrumento, como la guitarra, el acordeón, etc.), incorporación de hábitos de vida saludable y promoción de una salud positiva, etc.

A diferencia de lo anterior, dentro de contextos informales de aprendizaje (tales como crianza recibida por sus padres, relación con grupo de pares, el patio, la multicancha, etc.), han podido aprovechar sus experiencias previas y competencias, para adquirir valores como el respeto, la confianza, la solidaridad, la confianza, etc.; asumir y/o apoyar labores mantención de un hogar (limpieza y aseo pieza, lavar loza, barrer, cocinar, ordenar, etc.); manejo de un computador; valoración de los estudios, como mecanismo de superación y desarrollo personal; desarrollo de un mayor nivel de responsabilización (siendo capaz de hacer trámites como pagar servicios básicos, como luz o agua, etc.); cuidado y/o acompañamiento familiares significativos (en trámites de salud, cuidado sobrinos, etc.); conocimiento y manejo de un predio agrícola (asumiendo diversas funciones, tales como hacer leña y carbón; cuidado, crianza y faena de animales; etc.); visión positiva respecto al futuro, etc. Sin embargo, no todos los aprendizajes adquiridos dentro de este contexto han sido socialmente positivos, sino que también han podido desarrollar diversas funciones cognitivas (planificación, comparación, toma de decisiones, observación, etc.) en la ejecución de diversos delitos (judicializados o no), obteniendo dinero fácil para solventar actividades recreativas con grupo de pares (jugar pool, fútbol asistencia a fiestas por ejemplo), suplir carencias económicas (debido a que provienen de familias con bajos ingresos y escaso nivel escolar), hacer de la sobrevivencia un estilo de vida, velar por sus propias necesidades (pasando a llevar a otras personas); desarrollo de un alto nivel de liderazgo, validación e influencia frente a sus pares, etc.

En tercer lugar y refiriéndonos a las condiciones, contextos y situaciones en las cuales se manifiesta la propensión a aprender de los adolescentes infractores de ley, podemos afirmar que la propensión a aprender de estos adolescentes fluye de manera autoorganizada, libre y autónoma en todos aquellos espacios, situaciones o contextos informales de aprendizaje 
(patio, plaza de juegos, multicancha, áreas verdes, etc.), incluso aunque esta misma propensión movilice a los adolescentes hacia la comisión de la infracción de ley, dado que no existen lógicas arbitrarias y asimétricas de regulación del comportamiento. Lo anterior, nos permite mostrar cómo esta propensión a aprender muestra un alto nivel de desarrollo y estimulación en contextos informales, pero se vería dramáticamente restringida y anquilosada dentro de las formalidades del sistema escolar actual, donde se instaura hegemónicamente qué, cuándo y cómo se aprende, convirtiéndose este proceso en algo aburrido, monótono, difícil y complicado. Esta situación genera un profundo desencantamiento y rechazo hacia las lógicas de enseñanza - aprendizaje en la cual se escolarizan estos jóvenes, al verse forzados a incorporar linealmente una serie de contenidos que muchas veces no les son significativos o necesarios para sus vidas, aumentando las probabilidades del fracaso escolar.

En cuarto lugar, haciendo mención a las relaciones generadas por los adolescentes infractores de ley, a lo largo de sus trayectorias vitales que los han conducido a uno (o varios) episodio (s) de infracción de ley, podemos aseverar tras el análisis de las historias de vida de estos jóvenes, que la mayoría de ellos logran darse cuenta (con mayor o menos claridad), que su vinculación al delito se vio influida y/o potenciada por una serie de características que compartían con el grupo de pares que fueron formando (principalmente desde el paso de la enseñanza básica a la enseñanza media), entre las cuales se pueden mencionar que la pareja del adolescente y/o grupo de pares mostraban un alto compromiso delictual (diversas causas por hurto, robo, etc.); existencia de patrones de consumo de drogas con fines recreativos, comisión del delito como una forma de validación frente al grupo de pares y/o demostrar lealtad o compromiso frente a ellos etc.

En quinto lugar, y refiriéndonos a las alternativas de modificabilidad cognitiva descubiertas a través de esta investigación, para los adolescentes infractores de ley, podemos mencionar que la propensión a aprender que exhiben estos adolescentes demuestra la existencia de una amplia gama de recursos, habilidades y potencialidades presentes en estos jóvenes, los cuales no están siendo debidamente reconocidos y/o valorados dentro del sistema escolar actual. Así, hemos podido identificar algunas condiciones o elementos básicos que permitirían concretar la modificabilidad cognitiva cumpliendo entre otras cosas, con las siguientes características:

- Repensar la escuela por medio de la generación de experiencias de aprendizaje mediado.

- Existencia de la figura de un mediador (tal como un profesor, amigo, padre, etc.), en la vida de los adolescentes que sea capaz de identificar y potenciar sus recursos y potencialidades; teniendo una visión positiva de ellos (evitando la estigmatización), transmitirle confianza y afecto.

- Creación de ambientes activos - modificantes, donde se promueva 
la curiosidad, la exploración, la despreocupación del error, la mediación de significado y la reciprocidad. Es decir, estos adolescentes poseen una gran cantidad de recursos cognitivos que no logran aprovechar (debido a que muchas veces desconocen su propia propensión a aprender) dentro de contextos estructurados y formales como es la escuela, debido a que se les inculca desde temprana edad que no pueden aprender y que son incapaces de lograr algo. De manera que resulta vital poder propiciar un modelo educativo que estimule la libertad, la confianza (por medio de la conversación y la colaboración), la autonomía, el aprendizaje práctico (donde haya mayor poder de decisión acerca de lo que es necesario e importante saber); utilización significativa de las habilidades lingüísticas.

- Recibir apoyo y orientación permanente en la superación de aquellas experiencias de fracaso (tal como una repitencia escolar, vinculación a un episodio de infracción de ley, etc.).

- Intencionalidad / Orientación socio afectiva en la Construcción y Definición de un Proyecto de Vida en los adolescentes. 


\section{Notas}

${ }^{1}$ Este artículo presenta los resultados y hallazgos más relevantes surgidos de la investigación realizada por el autor, titulada "Construcción Socio-histórica de los Propensión a Aprender de los Adolescentes Infractores de Ley”. Tesis de Magíster en Educación, Mención Políticas y Gestión Educativa. Universidad Austral de Chile, Valdivia. Trabajo que se encuentra asociado al Proyecto FONDECYT N 1110577: “Asombros Educativos Infantiles y Propensión a Aprender”.

² Según Calvo (2008: 327), desde el nacimiento, el ser humano es pura propensión al aprendizaje. Simplemente aprende; no puede evitarlo, aunque quisiera. Así como no sabe que aprende, también ignora si tiene alguna dificultad para aprender, ya que todavía no le han enseñado que aprender es difícil, ni ha aprendido esa lección, tal vez una de las consecuencias más radicales en su vida. Quienes olvidan esta propensión a aprender, trivializan la enseñanza y la evaluación escolar.

${ }^{3}$ De acuerdo al SENAME (2008), el objetivo central de los Programas de Salidas Alternativas es desarrollar un programa de trabajo individualizado con adolescentes a quienes se les ha decretado una salida alternativa al procedimiento penal, que contemple una supervisión sistemática e intervenciones fundadas en un proceso de evaluación, y que contribuya al cumplimiento de la medida judicial y la integración efectiva a las redes locales.

${ }^{4}$ Introdujo algunas modificaciones importantes tales como el fin del examen de discernimiento para los adolescentes mayores de 16 años y menores de 18 años y baja la responsabilidad penal, que ahora es a partir de los 14 años. Adicionalmente, se cambia el enfoque de la justicia juvenil de un sistema coercitivo a uno que va orientado a la reinserción social de aquellos que han infringido la ley penal (SENAME, 2011). Para encontrar mayor información al respecto, sugerimos visitar el sitio web del Servicio Nacional de Menores, en la sección de Justicia Juvenil: www.sename.cl

${ }^{5}$ Reuven Feuerstein y Otros (1980), en su libro "Instrumental Enrichment: An Intervention Program for Cognitive Modifiability, distinguirían 2 tipos de determinantes: distales (correspondiente a aquellos factores genéticos, organicidad, nivel de estimulación ambiental, aspectos emocionales del niño y de sus padres, estatus socioeconómico, etc.) y aquellos proximales (descritos como aquella falta o insuficiencia de experiencias de aprendizaje mediado en el niño).

${ }^{6}$ El Atlas Ti es uno de los programas de análisis cualitativo más completo que existe en la actualidad, diseñado por Muhr (1991) en la Universidad Técnica de Berlín. Este paquete de apoyo a la investigación en ciencias sociales, aporta un conjunto de herramientas informáticas que permiten avanzar desde las simples tareas de codificación y recuperación hasta el punto final de construcción de teoría, herramienta coherente con la Teoría Fundada, como recurso metodológico idóneo en la presente investigación. Hemos consultado esta información, en el sitio web: http://www.atlasti.com/ uploads/media/atlas.ti6_brochure_2009_es.pdf 


\section{Bibliografía}

Andrews, D. y Bonta, J. (2006), The Psychology of Criminal Conduct. Anderson, Cincinnati.

Beloff, M. (2004), Los Derechos del Niño en el Sistema Interamericano. Del Puerto, Buenos Aires.

Bolívar, A. y Otros (2001), La Investigación Biográfico Narrativa en Educación. Enfoque y Metodología. La Muralla, Madrid.

Calvo, C. (2002), “Complejidad, Caos y Educación Informal”. En Revista de Ciencias de la Educación, № 190, Abril-Junio 2002. Instituto Calasanz de Ciencias de la Educación. pp. 227-245.

Ídem (2008), Del Mapa Escolar al Territorio Educativo. Disoñando la Escuela desde la Educación. Nueva Mirada, La Serena.

Ídem (2010), “Complejidades Educativas Emergentes y Caóticas”. En Revista Polis, Volumen 9, N 25, 2010. pp 87-100. Consultado en Junio 22, 2011 desde: <http://polis.revues.org/308>.

Calvo, C. y Elizalde, A. (2010), “Educación: Creación de Nuevas Relaciones Posibles”. En Revista Polis, Volumen 9, N²5, 2010. Universidad Bolivariana. pp. 7-15

Ezpeleta, L. (2005), Factores de Riesgo en Psicopatología del Desarrollo. Masson S. A., Barcelona.

Fernández, J. (2003), Delincuencia y Exclusión Social: Estructuras Sociales y Procesos de Socialización Imbricados. Documento de Trabajo Asesorías para el Desarrollo. Santiago de Chile. pp. 1-18

Feuerstein, R. (1983), La Teoría de la Modificabilidad Estructural Cognitiva. Mira S.A., Zaragoza.

Ídem (1991), “Mediated Learning Experience: A theoretical Review”. En Feuerstein, R. y Otros (1991), Mediated Learning Experience: Theoretical, Psychosocial, and Learning Implications. Freund Publishing House, Londres.

Feuerstein, R. y Otros (1998), Don't Accept me as I am. Helping Retarded People to Excel. Plenum Press, New York.

Flamey, G. (2006), La Deserción Escolar en Chile, ¿Prioridad en la Agenda Educativa?. Documento Foro Nacional Educación de Calidad para Todos. Asociación Chilena Pro Naciones Unidas, Santiago de Chile. pp. 1-25

Flick, U. (2004), Introducción a la Investigación Cualitativa. Morata, Ma- 
Polis, Revista Latinoamericana, Volumen 13, $N^{\circ}$ 37, 2014

drid.

Gómez, A. (2009), “Sujeción y Formación en la Educación Formal, No Formal e Informal”. En Revista de Investigación Educativa N ${ }^{\circ}$ 38, 2009. pp. 38-50. Consultado en Junio 25, 2011 desde: <http://www.educatio.ugto.mx/ PDFs/educatio7/Gomez.pdf $>$.

Foucault, M. (1996), Vigilar y Castigar. Siglo XXI, Madrid.

González, E. (1996), Menores en Desamparo y Conflicto Social. CCS, Madrid.

Gottfredson y Otros (1996), Delinquency. Handbook of adolescent health risk behavior. Plenum press, New York.

Hein, A. (2004), Factores de Riesgo y Delincuencia juvenil. Revisión de la Literatura Nacional e Internacional. Fundación Paz Ciudadana, Santiago de Chile.

Instituto Nacional de la Juventud (2007), Quinta Encuesta Nacional de la Juventud. Consultado en Junio 16, 2011 desde: <http://www.unicef.cl/unicef/ public/archivos_documento/283/WD_10.pdf >.

Lombaert, E. (1999), “Vulnerabilidad Societal”. En Revista El Observador, $\mathrm{N}^{\circ}$ 19. Servicio Nacional de Menores, Santiago de Chile. pp. 11-32

López de Maturana, S. (2010), Maestros en el Territorio. Universidad de La Serena. La Serena.

Mendicoa, G. y Otros (1999), Exclusión y Marginación Social. Espacio, Buenos Aires.

Montero, A. (2000), La Convivencia en los Centros escolares: Modelo de Intervención y Marco Normativo. Aljibe, Archidona.

Moreira, M. (1997), Aprendizaje Significativo: Un Concepto Subyacente. Consultado en Octubre 03, 2012 desde: <http://www.arnaldomartinez.net/ docencia_universitaria/ausubel03.pdf $>$.

Moreno, A. y Calvo, C. (2010), "Etnoeducación, Educación Física y Escuela: Transitando desde la Educación Informal a la Escuela Autoorganizada”. En Revista Ágora para la Educación Física y el Deporte, № 12 (2). MayoAgosto 2010. pp. 131-150.

Muñoz, L. (2008), Los Jóvenes y el Nuevo Sistema de Responsabilidad Penal Adolescente a un Año de su Implementación. En Serie Reflexiones Infancia y Adolescencia $N^{\circ}$ 10, Diciembre 2008. UNICEF, Santiago de Chile. 
Pineau, G. y Jobert, G. (1989), Histoires de Vie. Vol. 1: Utilisation pour la Formation. L`Harmattan, Paris.

Pineau, G. y Le Grand, J. (1993), Les Histoires de Vie. PUF, Paris.

Pujadas, J. (1992), El Método Biográfico: El Uso de las Historias de Vida en Ciencias Sociales. Centro de Investigaciones Sociológicas. Madrid.

Rogers, A. (2004), Looking Again at Non-formal and Informal Education. Towards a New Paradigm. Consultado en Junio 23, 2011 desde: <http:// www.infed.org/biblio/non_formal_paradigm.htm>.

Seale, C. (1999), The Quality of Qualitative Research. SAGE, Londres.

Strauss, A. y Corbin, J. (2002), Bases de la Investigación Cualitativa. Técnicas y Procedimientos para Desarrollar la Teoría Fundamentada. Universidad de Antioquia. Colombia.

Tsukame, A. (1996), “Delincuencia y Subcultura: Alcance y Origen de la Delincuencia Juvenil”. En Revista de Estudios Sociales No 89, Julio - Septiembre 1996. Corporación de Promoción Universitaria, pp- 167-209.

Ídem (2010), “Deserción Escolar, Reinserción Educativa y Control Social del Delito Adolescente”. En Revista de la Academia N 15, Primavera 2010. Universidad Academia de Humanismo Cristiano, Santiago de Chile. pp. 4159. ISSN 0717-1846.

Vasilachis, I. y Otros (2006), Estrategias de Investigación Cualitativa. Gedisa, Barcelona.

Weitzman, E. (2000), “Software and Qualitative Research”, en Denzin, N. y Lincoln, S. Handbook of Qualitative Research ( $2^{\circ}$ Edición). SAGE, Londres. pp. 803-820.

Willis, P. (1988), Aprendiendo a Trabajar. Como los Chicos de Clase Obrera Consiguen Trabajos de Clase Obrera. Akal, Madrid.

Recibido: 21.08.2013

Aceptado: 14.04.2014 